GRASAS Y ACEITES 72 (1)

January-March 2021, e388

ISSN-L: 0017-3495

https://doi.org/10.3989/gya.1031192

\title{
Preparation and characterization of oleogels with tallow and partially hydrolyzed tallow as organogelators
}

\author{
${ }^{\oplus}$ E. Keskin Uslu and ${ }^{\circledR}$ E. Y Y $11 m a z{ }^{\bowtie}$ \\ Çanakkale Onsekiz Mart University, Faculty of Engineering, Department of Food Engineering, 17020, Çanakkale, Turkey \\ ${ }^{\bowtie}$ Corresponding author: eyilmaz@comu.edu.tr
}

Submitted: October 28, 2019; Accepted: December 16, 2019; Published online: February 24, 2021

SUMMARY: The aim of this study was to evaluate the organogelation potential of tallow fat (TF) and partially hydrolyzed tallow fat (HTF) against saturated monoglyceride (MG) and a saturated monoglyceride + diglyceride mixture (MDG) as the organogelators. TF itself created oleogel at a $30 \%$ addition level, while HTF, MG and MDG oleogels were prepared at 10\% addition levels. Fatty acid composition data showed that the oleogel of HTF (HTFO) was quite similar to those of MG and MDG oleogels. Solid fat content, free fatty acidity and peroxide values were found to be in acceptable ranges for HTFO. Thermal properties, crystal morphology and X-ray diffraction patterns were also evaluated. Rheological analyses indicated that all oleogels had higher storage modulus $\left(G^{\prime}\right)$ than loss modulus $\left(G^{\prime \prime}\right)$. The time-sweep test showed that after applying higher shear rates, the gels re-formed at rest. Further, all oleogels maintained their gelled consistency until around $54{ }^{\circ} \mathrm{C}$. The results suggest that HTF could be a cheap, efficient, fast melting, safe and readily available organogelator.

KEYWORDS: Microstructure; Organogelator; Partially Hydrolozed Tallow; Rheology; Tallow

RESUMEN: Preparación y caracterización de oleogeles con sebo y sebo parcialmente hidrolizado como organogeladores. El objetivo de este estudio fue evaluar el potencial de la organogelación de grasa de sebo (GS) y grasa de sebo parcialmente hidrolizada (GSH) contra monoglicéridos saturados (MG) y mezcla de monoglicéridos + diglicéridos saturados (MDG) como organogelantes. Con la propia GS se creó un oleogel con un nivel de adición del 30\%, mientras que los oleogeles de GSH, MG y MDG se prepararon con niveles de adición del 10\%. Los datos de composiciones de ácidos grasos mostraron que el oleogel de GSH (OGSH) era bastante similar a los oleogeles de MG y MDG. El contenido de grasa sólida, la acidez grasa libre y los valores de peróxido se encuentran en rangos aceptables para OGSH. También se evaluaron las propiedades térmicas, la morfología del cristal y los patrones de difracción de rayos X. Los análisis reológicos indicaron que todos los oleogeles tenían un módulo de almacenamiento $\left(\mathrm{G}^{\prime}\right)$ mayor que el módulo de pérdida $\left(\mathrm{G}^{\prime \prime}\right)$. La prueba de barrido de tiempo mostró que después de aplicar velocidades de cizallamiento más altas, los geles se reformaron en reposo. Además, todos los oleogeles protegieron su consistencia gelificada hasta alrededor de $54{ }^{\circ} \mathrm{C}$ de temperatura. Los resultados han sugerido que GSH podría ser un organogelador barato, eficiente, de fusión aguda, seguro y fácilmente disponible.

PALABRAS CLAVE: Microestructura; Organogelador; Reología; Sebo; Sebo parcialmente hidrolizado

Citation/Cómo citar este artículo: Keskin Uslu E, Yilmaz E. 2021. Preparation and characterization of oleogels with tallow and partially hydrolyzed tallow as organogelators. Grasas Aceites 72 (1), e388. https://doi.org/10.3989/gya.1031192

Copyright: (C2021 CSIC. This is an open-access article distributed under the terms of the Creative Commons Attribution 4.0 International (CC BY 4.0) License. 


\section{INTRODUCTION}

To fulfill the need for trans-free, low saturated and structured (plastic consistency) fats for the food industry, research studies on oleogelation technology have been accelerated in the past decade. Oleogel is simply defined as an oil in continuous liquid phase with macroscopic dimensions that are permanent on a time scale with immobilized networks of self-assembled molecules called organogelators (Co and Marangoni, 2012; Singh et al., 2017; Patel, 2018). With this technology, edible fats with various hardness and melting properties could be produced without trans acid formation or major changes in fatty acids or minor component compositions. Depending on the kinds and amounts of organogelators that form the gel network, totally edible, safe, and sensorially acceptable oleogels can be prepared (Bot et al., 2009; Co and Marangoni, 2012; Patel and Dewettinck, 2016; Patel, 2018). Based on the preparation approach and organogelators involved, various types of oleogels were developed such as directly dispersed systems, emulsion templates, structured biphasic systems and oil sorption systems (Patel and Dewettinck, 2016; Singh et al., 2017).

Low molecular weight gelators (LMWG) including mono-, di-, and tri-acyl glycerides, various fatty acids and alcohols, hydroxylated fatty acids, lecithin, various waxes (beeswax, shellac, sunflower, candelilla, carnauba, rice bran waxes), wax esters, ceramides, sorbitan derivatives, phytosterols, $\gamma$-oryzanol + phytosterol, sucrose esters, some amphiphiles, amino acid derivatives, and polymeric type gelators (PG) including some food proteins (gelatin, $\beta$-lactoglobulin, zein, gluten, gliadin), some hydrocolloids (xanthan gum, gum arabic, locust bean gum), ethyl cellulose, other cellulose derivatives, some carbohydrate derivatives (modified chitin, waxy starch) and combined gelator systems have been researched extensively (Terech and Weiss, 1997; Patel and Dewettinck, 2016; Patel, 2018; Sagiri et al., 2018). New and more convenient (food-grade, cheap, readily available, effective at low concentrations, matching desired physical properties, versatile, and permitted) organogelators for edible applications are currently under investigation (Co and Marangoni, 2012; Patel, 2018).

It was stated that a material could act as an organogelator if it poses a balanced soluble and insoluble portion on the same molecule in the oil continuous media to trigger solute-solvent and solute-solute interactions simultaneously. Through molecular interactions via $\mathrm{H}$-bonding, van der Waals attractions and $\pi-\pi$ stacking between the phases, supramolecular structures like crystal lattice, liquid crystals, micelles, bilayers, fibrils, and agglomerates result in oleogelation (Rogers, 2009; Dassanayake et al., 2011; Patel, 2018).

Organogelators based on tri-, di, and monoglycerides or high melting point fractions of edible fats could provide the advantages of being food-grade, suitable for commercialization, cheap and easy to implement. Some examples of monoglyceride gels (Da Pieve et al., 2010; Ögütcü and Yilmaz, 2015), diglyceride gels (Rocha-Amador et al., 2014; Perez-Monterroza et al., 2014; Huang et al., 2018), triglyceride gels (Cerqueira et al., 2017), milk fat gels (Viriato et al., 2018), methyl cellulose coated palm stearine gels (Patel, 2017) exist in the literature. To the best of our knowledge, there has been no study on the applications of tallow fat or tallow fat-derived products used as organogelators. Since tallow is an edible and sensorially acceptable fat, it could be a feasible organogelator at possibly lower addition levels without significant enhancement of the saturated fatty acids.

Hence, the objective of this study was to evaluate the possibility of using tallow fat and partially hydrolyzed tallow fat in comparison with saturated monoglyceride and saturated monoglyceride + saturated diglyceride mixture as organogelators for structuring sunflower oil. The ultimate goal was to find cheaper, food-grade, efficient, and easily accessible new organogelators for edible applications.

\section{MATERIALS AND METHODS}

\subsection{Materials}

Refined-winterized sunflower oil (Biryağ, Trakya Birlik, Tekirdağ, Turkey), melted-filtered bovine body tallow fat (local store), saturated monoglyceride (Monomuls ${ }^{\circledR}$ 90-35 Saturated Monoglyceride, BASF Co., Illertissen, Germany), and a mono-palmitate + di-palmitate mixture (Veser Kimya, İstanbul, Turkey) were used in the study. All solvents and chemicals were of analytical grade and purchased either from Sigma Chem. Co. (St. Louis, MO, USA) or Merck (Darmstadt, Germany). 


\subsection{Partial hydrolysis of tallow fat}

A teflon-lined stainless steel autoclave $(100 \mathrm{ml})$ was used for tallow hydrolysis. Melted tallow fat:deionized water $(1: 2, \mathrm{v} / \mathrm{v})$ was put into the autoclave and then sealed. The autoclave was heated under an autogeneous pressure at $200{ }^{\circ} \mathrm{C}$ for $4 \mathrm{~h}$. After cooling down to room temperature, the partially hydrolyzed mixture was analyzed for free fatty acids (FFA) according to the method of Ca 5a-40 (AOCS, 1998). The FFA was $48.5 \%$, and the mixture was successfully washed with hot water (water: tallow, 1:1, v/v) until the FFA was reduced to $1.5 \%$. Although not used for any purpose in this study, the free fatty acids could be valorized as soapstock or animal feed. Then, the partially hydrolyzed tallow fat was crystallized at room temperature and washed several times with cold water. Finally, it was heated to $110{ }^{\circ} \mathrm{C}$ to evaporate all water before cooling down to ambient temperature to get the partially hydrolyzed tallow fat. The initial tallow fat and partially hydrolyzed tallow fat were used as the new organogelators in this study. Both were creamy-white, odorless, solid products.

\subsection{Preparation of the oleogels}

Oleogels were prepared by mixing known portions of the organogelators and sunflower oil (SO) at $60{ }^{\circ} \mathrm{C}$. First, the minimum gelling concentration (MGC) of tallow fat (TF) as the organogelator was determined by adding the serial proportion into the oil (SO:TF, from 95:5 to $60: 40, \mathrm{w} / \mathrm{w})$. It was determined that TF forms a stable gel at a 30\% addition level (SO:TF, 70:30, $\mathrm{w} / \mathrm{w}$ ). Below that level, the oleogel was not stable and semi-liquid. Similarly, the MGC of the partially hydrolyzed tallow fat (HTF) was determined as $10 \%$. The oleogels of monoglyceride and monoglyceride+diglyceride mixture were prepared at the same addition level $(10 \%)$ for comparison. For all oleogels, after preparing the SO and organogelator mixtures, the beakers were put into a water bath $\left(60^{\circ} \mathrm{C}\right)$ and shaken. After complete melting, the mixtures were put into plastic cups and tubes. After cooling to ambient temperature, the prepared oleogels stayed in a refrigerator overnight. The next day, the samples were taken from the refrigerator and kept at ambient temperature. After stabilizing $24 \mathrm{~h}$ at room temperature, the analyses were started. During the analyses, the oleogels were kept at ambient temperature as well. The following abbreviations are used thoroughout the paper for the prepared oleogels: tallow fat oleogel (at 30\% addition level) (TFO), partially hydrolyzed tallow fat oleogel (HTFO), monoglyceride oleogel (MGO), and monoglyceride+diglyceride mixture oleogel (MDGO).

\subsection{Fatty acid composition analysis}

The fatty acid compositions of sunflower oil (SO), tallow fat (TF), partially hydrolyzed tallow fat (HTF) and the oleogels prepared with tallow fat (TFO), hydrolyzed tallow fat (HTFO), monoglyceride (MGO), and mono- and diglyceride mixture (MDGO) organogelators were determined. Fatty acid methyl esters were prepared according to method Ce 2-66 (AOCS, 1998) and quantified by a Gas Chromatograph (Agilent Technologies 7890B, Palo Alto, CA, USA) equipped with a flame ionization detector (FID) (Agilent Technologies, Palo Alto, CA, USA), and HP 88 capillary column $(100 \mathrm{~m} \times 0.25 \mathrm{~mm}$ ID $\times 0.2 \mu \mathrm{m}$ film thickness, $\mathrm{J} \& \mathrm{~W}$ Scientific Co, CA, USA). The oven was set to $120^{\circ} \mathrm{C}$ for $1 \mathrm{~min}, 175^{\circ} \mathrm{C}\left(10{ }^{\circ} \mathrm{C} / \mathrm{min}\right)$ for $10 \mathrm{~min}$, $210{ }^{\circ} \mathrm{C} \quad\left(5{ }^{\circ} \mathrm{C} / \mathrm{min}\right)$ for $5 \mathrm{~min}$ and $230{ }^{\circ} \mathrm{C}$ $\left(5^{\circ} \mathrm{C} / \mathrm{min}\right.$ ) for $5 \mathrm{~min}$. One $\mu \mathrm{L}$ injection volume, 1:50 split ratio, $2 \mathrm{ml} / \mathrm{min}$ flow rate with $\mathrm{H}_{2}$ carrier gas, and 250 and $280{ }^{\circ} \mathrm{C}$ injector and detector temperatures, respectively, were used in the analysis. Fatty acid methyl esters were quantified by co-chromatography with FAME mixture standards (37-components, C4-C24, Supelco, Bellefonte, PA, USA).

\subsection{Physico-chemical analyses of the oleogels}

The gel formatin time (GFT) was determined by following the modified method of Dassanayake et al., (2009). First, the stock oleogels were melted completely in a water bath, and then $10 \mathrm{ml}$ of each melted oleogel were placed into glass tubes. The tubes were kept for $0.5 \mathrm{~h}$ at $70{ }^{\circ} \mathrm{C}$ in the water bath for temperature equilibration. All the tubes were taken from the water bath to ambient temperature (set to $20^{\circ} \mathrm{C}$ ) for gel formation. Meanwhile, the time elapsed was recorded. The tubes were then rotated $90^{\circ}$ and if no flow was observed, it was considered as formed gel and the time was recorded as GFT.

The method of Da Pieve et al., (2010) was adapted for the oil binding capacity (OBC) analysis. Empty Eppendorf tubes were weighed (a) and then $1 \mathrm{ml}$ of each completely melted organogel sample was placed into the tube, and left for $1 \mathrm{~h}$ for organogel formation in the 
refrigerator. After the formation of the oleogels, the tubes were carefully weighed (b) again and centrifuged at $9167 \mathrm{xg}$ for $15 \mathrm{~min}$ at $20^{\circ} \mathrm{C}$. Then, the tubes were turned over on a filter paper and left for $3 \mathrm{~min}$ for excess oil to drain. Finally, the tubes were weighed (c). The oil binding capacity (\% OBC) was calculated by the equation given below.

$$
\begin{aligned}
& \% \text { Released Oil }=\frac{(\mathrm{b}-\mathrm{a})-(\mathrm{c}-\mathrm{a})}{(\mathrm{b}-\mathrm{a})} \times 100 \text { and } \\
& \% \mathrm{OBC}=100 \text {-Released Oil }
\end{aligned}
$$

The solid fat content of the oleogels was determined following the ISO 8292 method (ISO, 2012) with a Bruker Nuclear Magnetic Resonance (NMR) spectrometer (The Minispec, Bruker Optics, Inc.). The measurements were performed at 20 and $35{ }^{\circ} \mathrm{C}$. Instrument calibrations were done with 0,31 and $73.5 \%$ solid fat containing standard solutions. The results were expressed as percent solid fat content.

The color of the organogel samples was measured on a Minolta CR-400 colorimeter (Konica Minolta Sensing, Osaka, Japan) with CIE lab standards, and the $\mathrm{L}, \mathrm{a}^{*}$ and $\mathrm{b}^{*}$ values were recorded.

The free fatty acids (FFA) and peroxide values $(\mathrm{PV})$ of the oils and oleogels were determined by the method of $\mathrm{Ca} 5 \mathrm{a}-40$ and $\mathrm{Cd} \mathrm{8-53} \mathrm{(AOCS,}$ 1998), respectively. FFA was calculated over the major fatty acid determined from the fatty acid composition analysis for each sample, and PV was given as miliequivalent $\mathrm{O}_{2}$ per $\mathrm{kg}$ sample.

\subsection{Thermal analysis of the oleogels}

The crystallization and melting onset and peak temperatures and enthalpies of the oleogel samples were measured with a Perkin-Elmer 4000 Series Differential Scanning Calorimetry (Groningen, The Netherlands). The instrument was calibrated with Indium and Zinc. 5-10 mg of oleogel samples were weighed into aluminum pans and sealed hermetically. The temperature program was set to heat from $20{ }^{\circ} \mathrm{C}$ to $100{ }^{\circ} \mathrm{C}$ at $10{ }^{\circ} \mathrm{C} / \mathrm{min}$; then cool the samples to $-30{ }^{\circ} \mathrm{C}$ at $10^{\circ} \mathrm{C} / \mathrm{min}$ and hold for $3 \mathrm{~min}$ at that temperature for full crystal formation and a final heating to $100{ }^{\circ} \mathrm{C}$ at $10{ }^{\circ} \mathrm{C} / \mathrm{min}$. The Pyris 1 Manager Software of the instrument was used for the calculations. All samples were analyzed at least in duplicate (Yilmaz et al., 2015).

\subsection{Polarized light microscopy of the oleogels}

Polarized light microscope pictures (PLM) of the oleogel samples were taken with an Olympus BX51 polarized light microscope (Olympus Optical Co., Ltd., Japan) with 10x ocular and 4x lens attached, and equipped with a CCD color video camera (Canon) at room temperature (Yilmaz et al., 2015).

\subsection{X-ray diffraction analysis of the oleogels}

X-ray diffraction (XRD) patterns of the oleogels were assessed with a PANalytical Empyrean model (The Netherlands) X-ray diffractometer. Radiation was applied at a scanning rate of $0.02 / 0.6(\mathrm{sec})$ within a $2.0-50^{\circ}$ (20) range under $45 \mathrm{kV}$ and $40 \mathrm{~mA} \mathrm{CuK \alpha}$ $(\lambda=1.54056 \AA)$. Data analysis was completed with X'Pert HighScore Plus software (Malvern Panalytical Ltd., Royston, UK) (Yilmaz et al., 2015).

\subsection{Rheological analyses of the oleogels}

Rheological analyses of the samples were performed with a DHR 2 rheometer (TA Instruments, USA) using plate-plate cross hatched geometry $(\Phi=40 \mathrm{~mm}$, gap $0.9 \pm 0.1 \mathrm{~mm})$. Temperature control was maintained with a Peltier system $\left( \pm 0.1{ }^{\circ} \mathrm{C}\right)$ under the lower plate. For each oleogel sample, amplitude sweeps (strain $=0.01$ $100 \%$ ) were performed at $10{ }^{\circ} \mathrm{C}$ with $1 \mathrm{~Hz}$ frequency to determine the linear viscoelastic region (LVR). After determining the strain values within the LVR, frequency sweep tests were performed for each sample at $10{ }^{\circ} \mathrm{C}, 0.02-0.26 \%$ strain range and frequencies from 0.1 to $100 \mathrm{~Hz}$, and the storage $\left(G^{\prime}\right)$ ve loss $\left(G^{\prime \prime}\right)$ moduli were determined. For amplitude, frequency and time sweep tests, the $10{ }^{\circ} \mathrm{C}$ constant temperature was selected because at that temperature, the oleogels were totally solid and comparison of their rheological behaviors was easier to make. Furthermore, temperature sweep tests were carried out to observe the effect of increasing temperatures on the flow behavior.

Time sweep tests for each sample were also done at $10{ }^{\circ} \mathrm{C}$ with $1 \mathrm{~Hz}$ frequency and strains in the LVR, and strains below $\left(\mathrm{LVR}_{\text {strain }} \geq\right.$ Strain $)$ and above $\left(\mathrm{LVR}_{\text {strain }} \leq\right.$ Strain) linear viscoelastic region. Time sweep was applied as $180 \mathrm{~s}$ for the first region, $180 \mathrm{~s}$ for the second region, and $900 \mathrm{~s}$ for the third region. 
Finally, a temperature ramp test was carried out from $0{ }^{\circ} \mathrm{C}$ to $60^{\circ} \mathrm{C}$ at $1{ }^{\circ} \mathrm{C} / \mathrm{min}$ at $1 \mathrm{~Hz}$ frequency in LVR. The soak time was 120 s. All samples were tested three times and the results were presented as the average values.

\subsection{Statistical analysis}

The production of the oleogels was replicated two times, and each replicate sample was analyzed at least twice for each test. The results were presented as mean values with standard deviations. The Analysis of Variance (ANOVA) with mean comparisons by Tukey's test was completed by Minitab v.16.1 software (Minitab, 2010). The level of confidence was set at $95 \%$.

\section{RESULTS AND DISCUSSION}

\subsection{Physico-chemical properties}

The common physico-chemical properties of the oleogels and stock fats are presented in Table 1. Gel formation times (GFT) of the oleogels were significantly different. While TFO formed gels after the longest period $(35 \mathrm{~min})$, the formation of MDGO took only $0.34 \mathrm{~min}$. In our previous studies (Yilmaz et al., 2015; Öğütcü and Y1lmaz, 2015), it was quite clear that different organogelator formed stable oleogels at various times. This outcome was expected since the crystalline network forming molecules were different. In MDGO and MGO, there were only mono-palmitate and mono- and di-palmitate mixtures; while in TF and HTF, there were diverse types of tri-, di-, and mono-glyceride mixtures.

The solid fat contents (SFC\%) of the oleogels were measured at 20 and $35{ }^{\circ} \mathrm{C}$ (Table 1). There were slight but significant differences among the samples. The highest SFC was determined in the TFO at both temperatures, while the lowest value was in the MGO sample. Since the same stock oil (SO) was used in the creation of the oleogel, this difference was attributed to the added organogelators. Also, while creating HTFO, MGO, and MDGO, only $10 \%$ of the gelators were added to create a stable gel; whereas $30 \%$ of tallow fat was added into the TFO to get the stable gel. Hence, this proportion difference may lead to the SFC difference. Since the main advantages of the oleogels were stated as the lower content of saturated fatty acids and zero content of trans fatty acids (Co and Marangoni, 2012; Patel, 2018), lower SFC is preferred in oleogels. In other words, if an organogelator provides no extra saturated or trans fatty acids, it is preferred. In this case, TF by itself was not a good organogelator since it formed stable oleogels only at the $30 \%$ addition level, at which the saturated fatty acid content of the resulting oleogel was also increased. Partially hydrolyzed tallow fat (HTF), on the other hand, yielded stable oleogel at a $10 \%$ addition level, at which the saturated fatty acid content of the resulting oleogel was not

TABLE 1. Physico-chemical properties of the oleogels developed

\begin{tabular}{|c|c|c|c|c|c|c|c|c|c|}
\hline & \multirow{2}{*}{$\begin{array}{l}\text { Gel Formation } \\
\text { Time (min) }\end{array}$} & \multirow{2}{*}{$\begin{array}{c}\text { Oil Binding } \\
\text { Capacity (\%) }\end{array}$} & \multicolumn{2}{|c|}{ SFC (\%) } & \multirow{2}{*}{ L Value } & \multirow{2}{*}{$a^{*}$ Value } & \multirow{2}{*}{ b* Value } & \multirow{2}{*}{$\begin{array}{l}\text { Free Fatty Acidity } \\
\text { (major fatty acid \%) }\end{array}$} & \multirow{2}{*}{$\begin{array}{c}\text { Peroxide Value } \\
\left.\text { (meq } \mathrm{O}_{2} / \mathbf{k g}\right)\end{array}$} \\
\hline & & & $20{ }^{\circ} \mathrm{C}$ & $35^{\circ} \mathrm{C}$ & & & & & \\
\hline SO & - & - & - & - & $56.19 \pm 1.1$ & $-3.07 \pm 0.2$ & $13.47 \pm 0.9$ & $0.56 \pm 0.0$ & $2.59 \pm 0.5$ \\
\hline $\mathrm{TF}$ & - & - & - & - & $90.06 \pm 0.2$ & $-3.23 \pm 0.0$ & $2.38 \pm 0.1$ & $1.69 \pm 0.1$ & $9.72 \pm 0.0$ \\
\hline HTF & - & - & - & - & $91.35 \pm 0.5$ & $-3.10 \pm 0.1$ & $1.75 \pm 0.5$ & $1.55 \pm 0.5$ & $11.50 \pm 1.4$ \\
\hline TFO & $35.00 \pm 1.0^{a^{*}}$ & $99.61 \pm 0.1^{\mathrm{a}}$ & $12.50 \pm 1.2^{\mathrm{a}}$ & $7.05 \pm 1.5^{\mathrm{a}}$ & $49.27 \pm 3.8^{\mathrm{b}}$ & $-3.77 \pm 0.2^{b}$ & $-1.22 \pm 0.5^{\mathrm{d}}$ & $0.85 \pm 0.0^{\mathrm{a}}$ & $13.57 \pm 1.5^{\mathrm{a}}$ \\
\hline HTFO & $33.30 \pm 1.5^{\mathrm{a}}$ & $99.60 \pm 0.0^{\mathrm{a}}$ & $10.11 \pm 1.5^{\mathrm{a}}$ & $4.87 \pm 0.8^{b}$ & $47.59 \pm 2.0^{\mathrm{bc}}$ & $-2.15 \pm 0.1^{\mathrm{a}}$ & $1.40 \pm 0.1^{\mathrm{c}}$ & $0.71 \pm 0.1^{\mathrm{b}}$ & $11.34 \pm 0.0^{\mathrm{b}}$ \\
\hline MGO & $8.33 \pm 0.5^{\mathrm{b}}$ & $99.99 \pm 0.0^{\mathrm{a}}$ & $8.12 \pm 1.2^{\mathrm{b}}$ & $4.33 \pm 0.9^{b}$ & $52.01 \pm 0.2^{\mathrm{b}}$ & $-2.52 \pm 0.0^{\mathrm{a}}$ & $10.12 \pm 0.1^{\mathrm{b}}$ & $0.56 \pm 0.0^{c}$ & $12.14 \pm 0.7^{\mathrm{a}}$ \\
\hline MDGO & $0.34 \pm 0.0^{c}$ & $99.90 \pm 0.0^{\mathrm{a}}$ & $9.30 \pm 0.7^{\mathrm{ab}}$ & $4.75 \pm 0.6^{\mathrm{b}}$ & $67.06 \pm 1.1^{\mathrm{a}}$ & $-3.10 \pm 0.1^{\mathrm{b}}$ & $12.06 \pm 0.6^{\mathrm{a}}$ & $0.56 \pm 0.0^{\circ}$ & $9.70 \pm 1.9^{c}$ \\
\hline
\end{tabular}

SO: sunflower oil, TF: tallow fat, TFO: tallow fat oleogel, HTFO: hydrolized tallow fat oleogel, MGO: monoglyceride oleogel, MDGO: monoglyceride + diglyceride oleogel *Small letters within each column indicate significant differences among the oleogel samples for the mean \pm SD values calculated from four determinations by one-way analysis of variance and Tukey's test $(\mathrm{p} \leq 0.05)$ 
significantly enhanced. Since it is cheaper, edible, legally permitted and sensorially acceptable, it might be a better alternative as organogelator.

The oil binding capacity (OBC) values of the oleogels were well above $99 \%$ for all samples, and not significantly different. This is a very important finding which indicates the presence of well-formed and stable gels. Clearly, with the organogelators used, almost all liquid oil was bound or entrapped. Quite similar results with wax gelators (Yilmaz et al., 2015), fatty acid and fatty alcohol gelators (Bot and Flöter, 2018), ethyl cellulose gelator (Mattice and Marangoni, 2018), and others (Toro-Vasquez et al., 2018; Patel, 2018) were found in the literature.

The instrumental color values of the samples were also measured (Table 1). The $\mathrm{L}$ values of SO and TF were significantly different, and melted tallow fat was quite luminous, but their a* values were not statistically different. The yellow color ( $b^{*}$ value) of SO was significantly different from $\mathrm{TF}$. When the oleogels were compared among themselves, the most luminous sample was MDGO. The $\mathrm{a}^{*}$ value (redness-greenness) was not significantly different among the oleogels; while the $b^{*}$ value (yellowness-blueness) was different. MDGO and MGO were yellow-toned samples; while the TFO sample had some blueness. The oleogels can be seen in Figure 1. It is well known from the oleogel literature that the color of an oleogel is mainly determined by the stock oils used and the color and addition level of the organogelators used (Patel, 2018).

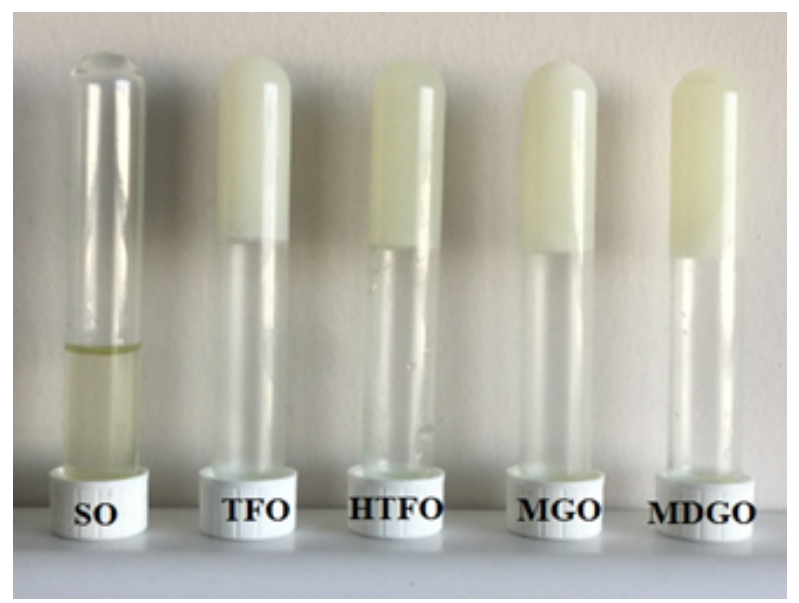

FIGURE 1. The stock oil (SO: sunflower oil) used in oleogel preparation, and the oleogels developed (TFO: tallow fat oleogel, HTFO: hydrolyzed tallow fat oleogel, MGO: monoglyceride oleogel, MDGO: monoglyceride + diglyceride oleogel)
Two chemical properties, free fatty acidity (FFA) and peroxide value (PV), were also measured (Table 1). Both values were determined in the stock liquid oil (SO), tallow fat (TF), and partially hydrolyzed tallow fat (HTF), which were used in oleogel preparations. Both values were higher in TF and HTF than those in the SO, but in all samples, the values were below the edible vegetable oil codex standards (Codex, 2017), which states $0.6 \mathrm{mgKOH} / \mathrm{g}$ acid value and $10 \mathrm{meqO}_{2} / \mathrm{kg} \mathrm{PV}$ as limit values. When the oleogels were compared among themselves for FFA value, TFO and HTFO had slightly higher $(0.85$ and $0.71 \%)$ values than those of the MGO and MDGO $(0.56 \%)$, respectively. This was an expected result since the mono- and di-glycerides used as gelators were pure substances. But all oleogel samples were fairly acceptable for FFA according to the edible oil codex. On the other hand, except for the MDGO sample $\left(9.70 \mathrm{meqO}_{2} /\right.$ $\mathrm{kg}$ ), the PV of all samples exceeded the codex limit. This might be due to the presence of heat and air during oleogel preparation, which might have caused oil oxidation. Hence, vacuum or nitrogen atmospher and antioxidant addition could be suggested during oleogel production like all other oleogels reported in the literature.

The fatty acid composition of the oils/fats used as stock materials and the prepared oleogels were also measured, and the results are presented in Table 2. Four main fatty acids in the SO and five main fatty acids in TF were determined. Both composition data mainly concur with the current literature (Codex, 2017; Love, 1996). After the partial hydrolysis and consequent washing of the free fatty acids of the TF, the HTF was obtained, and its fatty acid composition analysis indicated the presence of three fatty acids. Compared to TF, the proportion of palmitic acid was enhanced significantly $(77.95 \%)$ in the HTF; while myristic and linoleic acids could not be quantified. Further, significant decreases in the stearic and oleic acids were quantified in HTF. When the oleogels were compared, it was observed that the total saturated fatty acid contents of TFO (22.50\%), MGO (26.66\%) and MDGO (33.89\%) were significantly higher than that of the HTFO $(15.60 \%)$. This could be considered as a promising result in terms of nutritional perspective. Also, it is clear that the content of total saturated fatty acids is not the only factor governing oleogel formation. In fact, the presence of non-polar and polar groups on the same molecule like mono- and di-glycerides made them more efficient as organogelator 
Preparation and characterization of oleogels with tallow and partially hydrolyzed tallow as organogelators $\bullet$

TABLE 2. Fatty acid compositions of the oil/fat used and the oleogels prepared

\begin{tabular}{lccccccc}
\hline Fatty Acid & SO & TF & HTF & TFO (30\%) & HTFO (10\%) & MGO (10\%) & MDGO (10\%) \\
\hline C14:0 & - & $3.34 \pm 2.50$ & - & $0.90 \pm 0.20$ & - & - & - \\
C16:0 & $4.46 \pm 1.30$ & $30.65 \pm 2.40$ & $77.95 \pm 1.50$ & $12.50 \pm 1.50$ & $11.80 \pm 1.40$ & $24.11 \pm 1.02$ & $29.15 \pm 3.23$ \\
C18:0 & $2.30 \pm 0.20$ & $23.40 \pm 0.80$ & $13.30 \pm 1.20$ & $9.10 \pm 1.72$ & $3.80 \pm 0.80$ & $2.55 \pm 0.50$ & $4.74 \pm 0.65$ \\
C18:1 & $36.24 \pm 1.55$ & $38.75 \pm 2.30$ & $8.75 \pm 0.50$ & $35.07 \pm 1.70$ & $33.90 \pm 1.90$ & $26.52 \pm 1.39$ & $28.72 \pm 1.60$ \\
C18:2 & $57.00 \pm 1.00$ & $3.05 \pm 1.00$ & - & $38.51 \pm 1.50$ & $50.50 \pm 1.50$ & $46.15 \pm 2.33$ & $36.94 \pm 1.67$ \\
$\Sigma$ Saturated & 6.75 & 57.39 & 77.95 & 22.50 & 15.60 & 26.66 & 33.89 \\
$\Sigma$ Unsaturated & 93.24 & 41.80 & 22.05 & 73.58 & 84.40 & 72.67 & 65.66 \\
\hline
\end{tabular}

SO: sunflower oil, TF: tallow fat, TFO: tallow fat oleogel, HTFO: hydrolized tallow fat oleogel, MGO: monoglyceride oleogel, MDGO: monoglyceride + diglyceride oleogel

(Patel, 2018). HTFO, MGO, and MDGO had only $10 \%$ organogelator added, but TFO had $30 \%$ organogelator added to obtain oleogels. Hence, TFO could not be accepted as oleogel, but rather it could be claimed as a blend of sunflower oil and tallow fat. Further, there was no beefy flavor or any off odor in TFO and HTFO. Overall, partially hydrolyzed tallow fat could be an effective organogelator compared to pure commercial mono- and di-glycerides. Since it did not enhance saturated fatty acid content significantly, and it is a much cheaper and more readily available material, this proposed partial hydrolysis mechanism could be used to obtain an organogelator to be applied in foods.

\subsection{Thermal properties}

The thermal properties of oleogels are very important quality parameters to evaluate their suitability for edible applications. It was indicated that melting and crystallization temperatures and ranges are one of the most common quality parameters for commercial margarine, shortenings, and other semi-solid fat products (Chrysam, 1996). Different product types (table spreads, kitchen margarines, tube margarine, shortenings, cake/batter fats, etc.) are expected to have certain thermal behaviors. Furthermore, there is a usually a well-documented correlation between the thermal behavior and SFC of the known commercial solid/semi-solid fat products. High quality spreads or kitchen margarines are expected to melt quickly in the mouth to yield a cooling effect with no lingering greasiness (O'Brien, 2008; Chrysam, 1996). Oleogels could be used to prepare margarine-like products and shortenings, and could even be applied themselves as spreadable fats (Öğütcü and
Y1lmaz, 2015). As seen in Table 1, it was obvious that oleogel products did not have high SFC, as expected, but they certainly had plastic consistency at ambient temperature. This situation is the-well known (Co and Marangoni, 2012) main advantage of oleogels. Hence, it is essential to determine the crystallization and melting ranges, peak temperatures and ethalpies. The thermal cycling technique (Dassanayake et al., 2009; Öğ̈ütcü and Y1lmaz, 2015) was used to get the full range of thermal behavior for both the organogelators used and the oleogels produced (Table 3).

The crystallization and melting onset and peak temperatures of the sunflower oil (SO) as stock oil for the gels, tallow fat (TF), partially hydrolyzed tallow fat (HTF), monoglyceride (MG), mono+diglyceride (MDG) as the organogelators, and the oleogels produced are presented in Table 3. SO, started to crystallize at $-16{ }^{\circ} \mathrm{C}$, and TF started at $11.67{ }^{\circ} \mathrm{C}$. Similarly, these two organogelators had peak melting temperatures of 28.66 and $15.69{ }^{\circ} \mathrm{C}$, respectively. Both $\mathrm{MG}$ and MDG had two fractions within themselves with different crystallization and melting temperatures, and both samples were similar in their thermal values. All oleogel samples (TFO, HTFO, MGO, MDGO) had two fractions presenting different thermal ranges. For crystallization and peak melting temperatures, HTFO was more similar to MGO and MDGO than to TFO. The peak melting temperatures of MGO and MDGO were 54.56 and $54.83{ }^{\circ} \mathrm{C}$ for the main fraction and 13.57 and $16.25{ }^{\circ} \mathrm{C}$ for the minor fraction, respectively. HTFO showed values of 12.39 and $47.11{ }^{\circ} \mathrm{C}$ for peak melting temperature. Clearly, HTFO totally melted at around $47{ }^{\circ} \mathrm{C}$, which seemed suitable for food product applications. The crystallization and melting profiles presented in Table 3 showed 
TABLE 3. Thermal properties of the oil/fat used and the oleogels prepared

\begin{tabular}{|c|c|c|c|c|c|c|}
\hline \multicolumn{4}{|c|}{ Crystallization } & \multicolumn{3}{|c|}{ Melting } \\
\hline & Onset $_{c}\left({ }^{\circ} \mathrm{C}\right)$ & Peak $\left(\mathrm{Tc},{ }^{\circ} \mathrm{C}\right)$ & $\Delta \mathrm{Hc}(\mathrm{J} / \mathrm{g})$ & Onset $_{\mathrm{m}}\left({ }^{\circ} \mathrm{C}\right)$ & $\operatorname{Peak}\left(\mathrm{Tm},{ }^{\circ} \mathrm{C}\right)$ & $\Delta \mathrm{Hm}(\mathrm{J} / \mathrm{g})$ \\
\hline SO & $-16.00 \pm 0.86$ & $-17.89 \pm 0.40$ & $-1.90 \pm 0.27$ & $22.89 \pm 1.11$ & $28.66 \pm 2.06$ & $7.05 \pm 0.10$ \\
\hline MG-Fr.1 & $11.93 \pm 0.27$ & $9.67 \pm 0.24$ & $-19.50 \pm 1.73$ & $10.39 \pm 0.28$ & $13.86 \pm 0.23$ & $23.93 \pm 1.87$ \\
\hline MG-Fr.2 & $63.69 \pm 0.17$ & $60.47 \pm 0.34$ & $-101.66 \pm 0.16$ & $61.68 \pm 0.13$ & $66.90 \pm 0.23$ & $105.45 \pm 0.98$ \\
\hline MDG-Fr.1 & $15.83 \pm 0.76$ & $14.11 \pm 1.18$ & $-15.74 \pm 1.11$ & $13.28 \pm 0.35$ & $16.21 \pm 0.43$ & $17.08 \pm 0.94$ \\
\hline MDG-Fr.2 & $65.79 \pm 0.08$ & $63.04 \pm 0.41$ & $-88.38 \pm 2.23$ & $63.52 \pm 0.26$ & $68.28 \pm 0.41$ & $82.77 \pm 5.57$ \\
\hline TF-Fr.1 & $11.67 \pm 1.90$ & $7.16 \pm 2.88$ & $-10.28 \pm 1.29$ & $15.70 \pm 2.66$ & $15.69 \pm 0.42$ & $15.83 \pm 2.40$ \\
\hline TF-Fr.2 & $31.45 \pm 2.76$ & $28.30 \pm 3.46$ & $-17.76 \pm 0.50$ & $34.08 \pm 0.69$ & $43.61 \pm 1.47$ & $29.92 \pm 0.47$ \\
\hline HTF-fr1 & $33.98 \pm 0.53$ & $30.44 \pm 0.03$ & $-10.15 \pm 0.04$ & $6.63 \pm 3.15$ & $12.89 \pm 1.89$ & $19.34 \pm 1.06$ \\
\hline HTF-fr2 & $56.19 \pm 0.71$ & $52.75 \pm 1.25$ & $-33.48 \pm 0.06$ & $57.27 \pm 0.84$ & $48.56 \pm 1.68$ & $30.07 \pm 0.71$ \\
\hline TFO-Fr.1 & $-0.56 \pm 0.10$ & $-3.33 \pm 0.08$ & $-2.92 \pm 0.52$ & $13.58 \pm 1.82$ & $14.37 \pm 0.69$ & $3.17 \pm 0.02$ \\
\hline TFO-Fr.2 & $22.77 \pm 0.69$ & $20.75 \pm 1.19$ & $-0.96 \pm 0.24$ & $33.36 \pm 1.44$ & $37.54 \pm 0.93$ & $25.37 \pm 0.69$ \\
\hline HTFO-Fr.1 & $11.05 \pm 0.09$ & $9.49 \pm 0.18$ & $-0.64 \pm 0.02$ & $9.67 \pm 0.03$ & $12.39 \pm 0.01$ & $3.23 \pm 0.20$ \\
\hline HTFO-Fr.2 & $44.19 \pm 2.25$ & $42.03 \pm 1.88$ & $-2.43 \pm 0.35$ & $32.90 \pm 0.71$ & $47.11 \pm 2.07$ & $2.31 \pm 0.41$ \\
\hline MGO-Fr.1 & $13.21 \pm 0.27$ & $9.32 \pm 1.24$ & $-1.86 \pm 0.35$ & $11.21 \pm 0.06$ & $13.57 \pm 0.95$ & $1.72 \pm 0.08$ \\
\hline MGO-Fr.2 & $52.22 \pm 0.76$ & $49.45 \pm 0.70$ & $-5.11 \pm 1.63$ & $47.02 \pm 0.40$ & $54.65 \pm 0.12$ & $5.76 \pm 2.12$ \\
\hline MDGO-Fr.1 & $15.76 \pm 0.13$ & $14.35 \pm 0.35$ & $-1.40 \pm 0.45$ & $14.47 \pm 0.32$ & $16.25 \pm 0.01$ & $1.73 \pm 0.68$ \\
\hline MDGO-Fr.2 & $52.74 \pm 0.86$ & $49.43 \pm 0.23$ & $-6.28 \pm 1.80$ & $43.79 \pm 1.24$ & $54.83 \pm 0.35$ & $7.26 \pm 0.22$ \\
\hline
\end{tabular}

SO: sunflower oil, MG: monoglyceride, MDG: monoglyceride + diglyceride, TF: tallow fat, HTF: partially hydrolyzed tallow fat, TFO: tallow fat oleogel, HTFO: hydrolized tallow fat oleogel, MGO: monoglyceride oleogel, MDGO: monoglyceride + diglyceride oleogel, Fr.1: fraction 1, Fr.2: fraction2

that HTFO could be used in margarine or spread formulations, like the monoglyceride oleogels used previously (Da Pieve et al., 2010; Ögütcü and Yilmaz, 2015). The SFC data presented in Table 1 for HTFO was consistent with DSC data where the sample had only around $5-10 \%$ solid fat at 25 to $35^{\circ} \mathrm{C}$, but was in solid (gelled) consistency, and completely melted at around $47{ }^{\circ} \mathrm{C}$. The difference between melting onset and peak temperatures showed the melting range, and HTFO had a melting range of around 1015 degrees, like MGO and MDGO. On the other hand, the peak melting temperature of HTFO $\left(47.11{ }^{\circ} \mathrm{C}\right)$ was lower than those of the MGO $\left(54.65{ }^{\circ} \mathrm{C}\right)$ and MDGO $\left(54.83{ }^{\circ} \mathrm{C}\right)$. For margarine and similar products, a narrower melting range closer to body temperature is preferred in order to produce a cooling sensation in the mouth to help taste buds perceive food flavor (Chrysam, 1996). In this respect, HTFO seems a better candidate for edible applications.

\subsection{Microstructural properties}

The polarized light microscopy (PLM) images of the oleogels are presented in Figure 2. Clearly, the crystalline structures of TFO and HTFO were similar but they were different from MGO and MDGO. In fact, all images indicated the presence of some aggregates, but the aggregates were quite large in MGO and MDGO. In a previous study (Öğütcü and Y1lmaz, 2015), hazelnut oil oleogels prepared with monoglyceride showed spherulitic or rosette-like crystalline patterns. Further, Kesselman and Shimoni (2007) published spherulitic crystals for corn oil-monoglyceride oleogels. The results confirmed that MGO and MDGO had spherulitic aggregate type crystals. In one study (Viriato et al., 2018), anhydrous milk fat was used as the organogelator to produce sunflower oil oleogels. Their PLM studies indicated crystals with long, fine needles of the spherulite type. Generally speaking, the crystal structure of an oleogel depends on the kind, purity and addition level of the organogelator, as well as the type of stock oil and process conditions (cooling rate, shear application, presence of other additives, etc.) as discussed (Mattice and Marangoni, 2018). In this study, the small, fine crystalline aggregates observed in the HTFO (Figure 2b) sample indicated that amphiphilic diand mono-glyceride mixtures present in the HTF 
could provide enough junction zones within the non-polar oil medium to yield the crystal lattice, where the mobile liquid oil was trapped. There were some aggregates in the TFO as well (Figure 2a), but since these were most probably the crystals of solid tallow triglycerides, they may not provide enough network (due to the absence of junction zones) to trap liquid oil at the $10 \%$ addition level, but could only provide enough solid surface for the oil to adhere at the $30 \%$ addition level. Overall, the partial hydrolysis of solid fat to provide higher melting fractions of diand mono-glyceride mixtures might be quite helpful for creating potential organogelators.
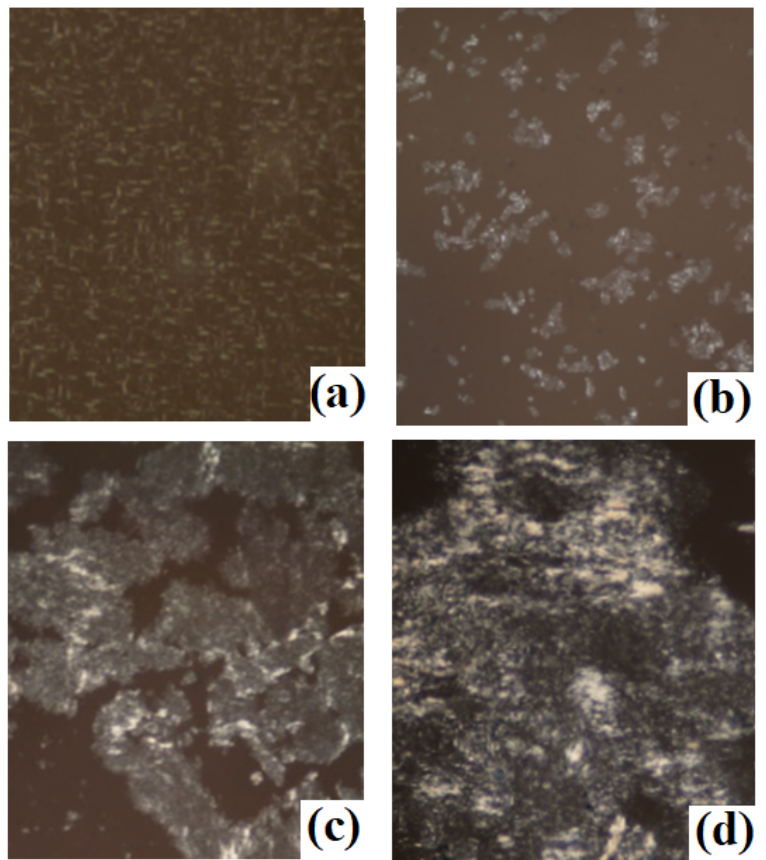

FigurE 2. The polorized light microscopy images of the oleogels with 40-fold magnification (a: TFO, b: HTFO, c: MGO and d: MDGO)

The X-ray diffraction patterns of the oleogels are presented in Figure 3. The wide angle region peaks of all four samples were quite similar to each other and were around 4.46-4.67 $\AA$ and 3.89-3.95 $\AA$, respectively. It was discussed (Stahl et al., 2017) that short spacings between fatty acid chains in solid lipids were in the $\alpha$-form if they were around $4.15 \AA$, and the $\beta$ ' form were around 3.8-4.2 $\AA$. Hence, the oleogels could be the $\beta$ ' type polymorph. Furthermore, small angle peaks at around 19.21-19.47 $\AA$ were found in the samples. There was a peak at around $43.29 \AA$ for only the MDGO sample, which might indicate the interplanar distance between the mono- and di- glyceride layers with a lamella of $43 \AA$. The data indicated that all crystallines in the oleogel samples could be $\beta$ ' polymorphs with an orthorhombic-perpendicular subcell $\left(\mathrm{O}_{\perp}\right)$ structure. Similar findings were reported for cod liver oil-monoglyceride organogels (Da Pieve et al., 2010) and wax organogels (Szydłowska-Czerniak et al., 2005). In addition, visual inspection by eye revealed that the oleogels were very smooth, homogeneous and creamy in texture (Figure 1).

\subsection{Rheological properties}

All rheological measurements were carried out within the linear viscoelastic region (LVR), beyond which the structure of the sample was destroyed. To determine the LVR, each sample was first tested by an amplitute sweep test at $10{ }^{\circ} \mathrm{C}, 1 \mathrm{~Hz}$ frequency with $0.01-100 \%$ strain. The LVR strain values determined were $0.26,0.16$, 0.11 and $0.023 \%$ for TFO, HTFO, MGO and MDGO, respectively. The amplitute sweep tests also indicated that the gel structure could be destroyed at around $8,50,50$, and $5 \mathrm{~Pa}$ oscillatory shear strain values for TFO, HTFO, MGO and MDGO, respectively. At those shear strain values, the cross-over points were reached, at which the gelled consistency was lost.

A frequency sweep test was performed within the LVR region at $10{ }^{\circ} \mathrm{C}$, and frequencies from 0.1 to $100 \mathrm{~Hz}$. In this test, oscillation frequency increased stepwise while keeping the amplitute constant. Frequency sweep tests can help to understand the time-dependent behavior of a sample in the non-destructive deformation range. The results of the measurements are presented in Figure 4. For all four samples, and within all the frequency ranges, storage moduli $\left(\mathrm{G}^{\prime}\right)$ were always higher than those of the loss moduli $\left(\mathrm{G}^{\prime \prime}\right)$. This means that during the storage period, the gel structure was stable in all samples. Phase segregation or settling should not develop during the storage period of these oleogels. Further storage modulus $\left(\mathrm{G}^{\prime}\right)$ values of HTFO were between 3.000-5.000 $\mathrm{Pa}$ throughout the applied angular frequency region of $0-1000 \mathrm{rad} / \mathrm{s}$ and were significantly higher than those of the TFO sample, which were between 100-300 Pa. This indicates that HTFO had a stronger gel structure. This situation is probably the result of a network formation by the amphiphilic mono- and diglycerides present in the HTF. Within the oil medium, amphiphilic molecules could create 

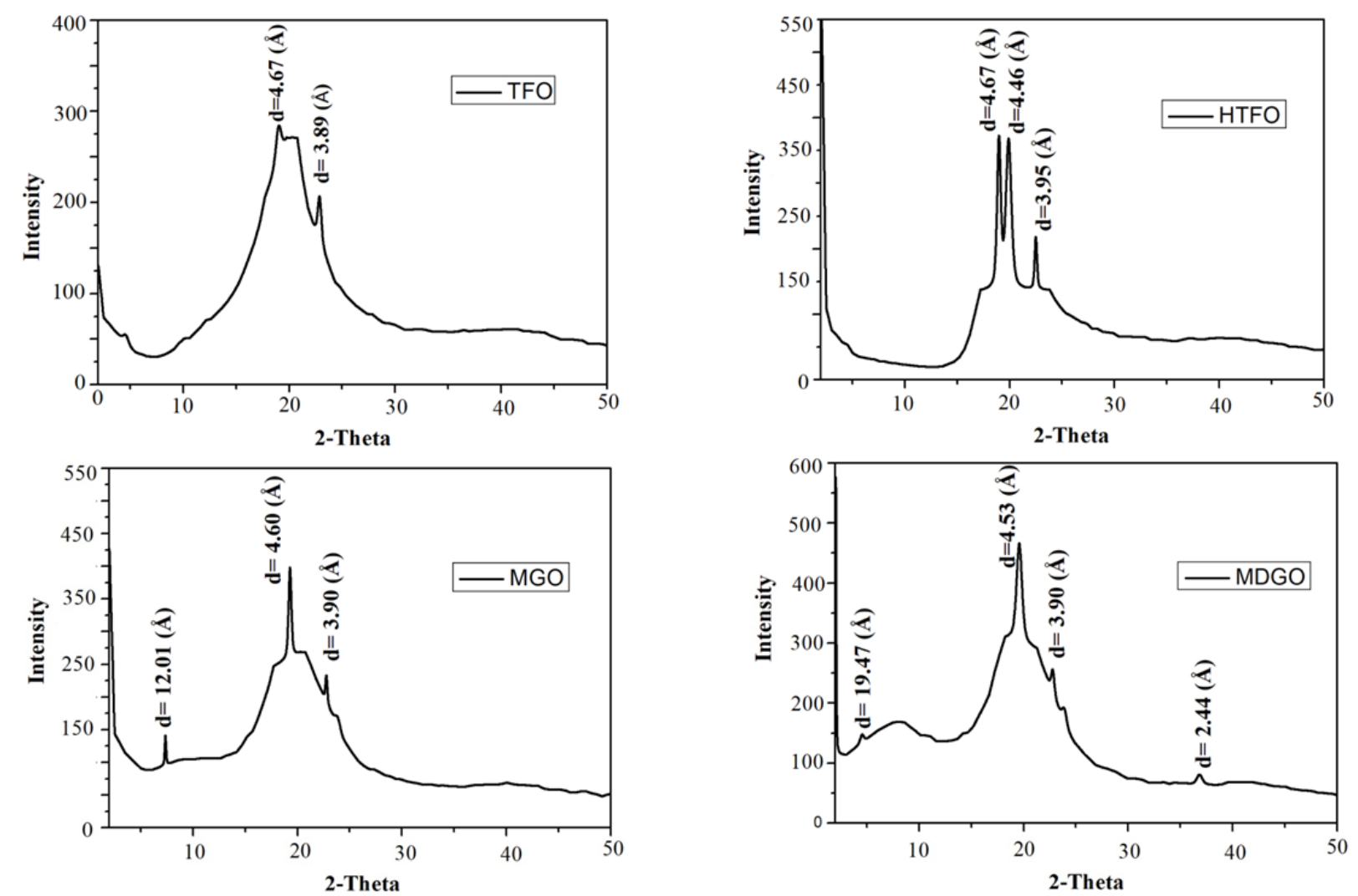

Figure 3. The X-Ray diffraction patterns of the oleogel samples

some junction zones for thermodynamic stability, and this yields the network that traps the liquid oil. Since TF forms oleogel due to its higher melting point triglycerides only, its gel is less strong than the HTF gel. When the storage modulus $\left(\mathrm{G}^{\prime}\right)$ of HTFO (around $3.000 \mathrm{~Pa}$ ) is compared to the storage modulus $\left(\mathrm{G}^{\prime}\right)$ values of MGO (around 80.0000 Pa) and MDGO (around $110.000 \mathrm{~Pa}$ ), it could be clearly observed that MGO and MDGO are much stronger gels. Overall, saturated mono- and di-glycerides were much better organogelators than saturated triglycerides. Hence, any solid fat stock could be partially hydrolyzed to its mono- and diglycerides to get an effective organogelator.

In order to observe the time-dependent viscoelastic behavior of the oleogel samples, time-sweep tests were performed with shearing under dynamic mechanical conditions, or under constant amplitute and frequency $(1 \mathrm{~Hz})$ at $10^{\circ} \mathrm{C}$. Three time regions were tested with preset oscillation so as to simulate the behavior at rest (first region). A strain at LVR was applied with 1 $\mathrm{Hz}$ frequency for $180 \mathrm{sec}$ to simulate structural breakdown or stronger shear. A strain higher than
$\operatorname{LVR}\left(\mathrm{LVR}_{1}>>\right)$ was applied with $1 \mathrm{~Hz}$ frequency for $180 \mathrm{sec}$, and finally to simulate structural regeneration at rest, a very low shear (LVRs $<<$ ) was applied with $1 \mathrm{~Hz}$ frequency for $900 \mathrm{sec}$. The results of the measurements are presented in Figure 5. In all samples at first region (very low shear to simulate resting behavior), the storage modulus $\left(\mathrm{G}^{\prime}\right)$ was higher than the loss modulus $\left(G^{\prime \prime}\right)$, and this indicates that all samples are gel structured. Once a strong shear was applied in the second region (simulate structural breakdown), both moduli decreased significantly as expected; hence, enough deformation occurred. Afterwards, in the third regeneration at the rest region with very low shear, both moduli increased again to their original levels observed in the first region. This means that a structural reformation or regeneration developed. Hence all four samples are truly gel, and all have mechanical regeneration ability. For oleogels, this ability is very important, and during product applications, for example, food formulation mixing processes, the deformed structure regenerated itself once mechanical force was removed. Similar thixotropic recovery was shown for shellac oleogels (Patel and Dewettinck, 2015). 


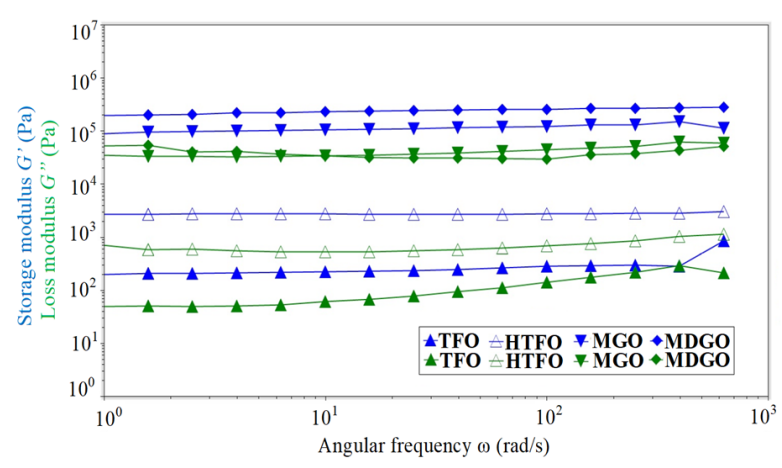

FIGURE 4. The frequency sweep test results of the oleogels developed (TFO: tallow fat oleogel, HTFO: hydrolized tallow fat oleogel, MGO: monoglyceride oleogel, MDGO: monoglyceride + diglyceride oleogel)

Lastly, a temperature-ramp test was carried out to observe the viscoelastic behavior under constant amplitute and frequency (Figure 6). All samples kept their gelled structure until the $\mathrm{G}^{\prime}$ and $\mathrm{G}^{\prime \prime}$ crossed at around $40{ }^{\circ} \mathrm{C}$ for $\mathrm{TFO}, 60{ }^{\circ} \mathrm{C}$ for HTFO, $63{ }^{\circ} \mathrm{C}$ for $\mathrm{MGO}$ and $61{ }^{\circ} \mathrm{C}$ for the MDGO sample. The peak melting temperature values of the same samples by DSC (Table 3) were 37.54, $47.11, \quad 54.65$ and $54.83{ }^{\circ} \mathrm{C}$, respectively. Obviously, peak melting temperatures were lower than those of the temperatures measured at the cross-over point (Figure 6). This might be due to the applied force during the rheologic measurement. There probably were some junction zones in the oleogel samples even after melting, at which the rheometer could still recognize some strength until the cross-over point reached at slightly higher temperatures. Clearly, HTFO was more stable until higher temperatures than TFO and better resembled MGO. This finding indicated that the partial hydrolysis of tallow fat yielded better properties as an organogelator. Both TFO and HTFO started to soften at around 20 and $30{ }^{\circ} \mathrm{C}$, but retained their gelled consistency until around 40 and $60{ }^{\circ} \mathrm{C}$, respectively. Although these temperatures were lower than those of the MGO and MDGO samples, they could still provide enough thermal stability for food applications.

\section{CONCLUSIONS}

Finding new, cheaper, readily available, safe and sensorially acceptable organogelators to initiate commercial production of oleogel products is still a research challange. In this context, tallow fat (TF) and partially hydrolyzed tallow fat (HTF) were investigated as organogelators in this study. They were compared to saturated monoglyceride and mono- and diglyceride mixtures. The results showed that HTF could be a very reliable organogelator for commercial applications. It yielded stable gels at a $10 \%$ addition level without extensively enhancing saturated fatty acid contents. In contrast, TF could create oleogel at a $30 \%$ addition level, at which it can no longer be considered as an oleogel, but rather as an oil-fat blend. Furthermore, the microstructure and rheological data of the HTF oleogel were quite similar to those of the MDGO and MGO samples. HTFO showed enough stability at applied strains and frequencies, just similar to the MGO and MDGO samples. HTFO showed enough structural recovery and thermal stability abilities to be used as an organogelator for food applications. Hence, abundantly available, cheap and safe organogelators could be created with a simple partial hydrolysis process from tallow fat. This study also investigated other similar hard fat sources to be evaluated as organogelators after partial glyceride hydrolysis.
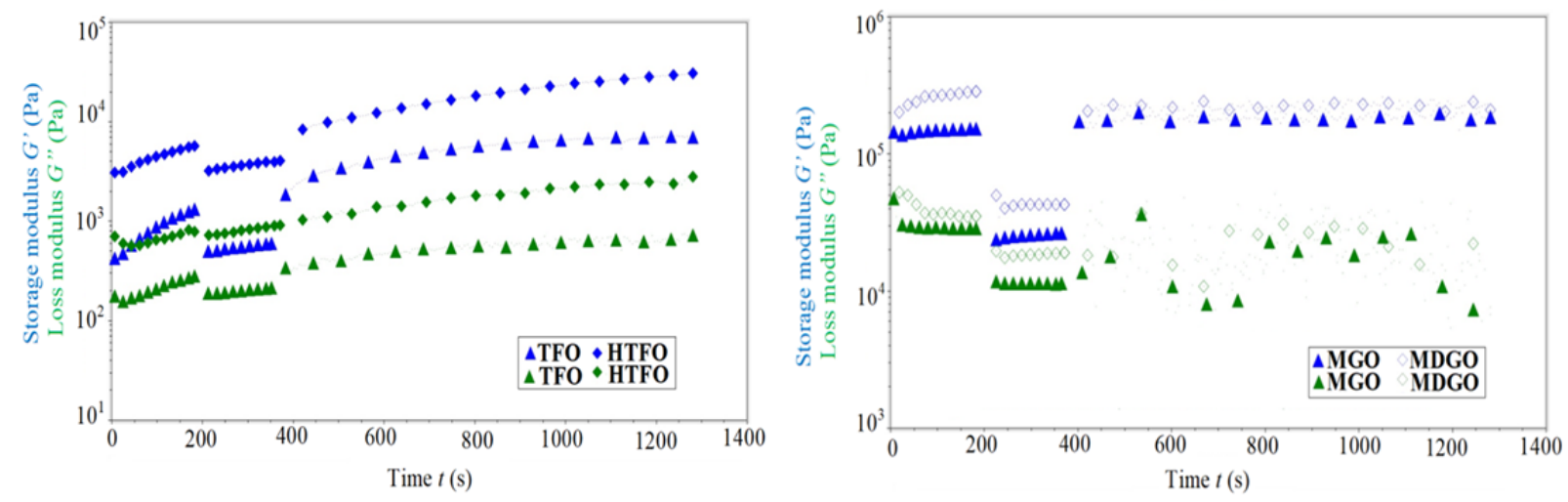

FIGURE 5. The time sweep test results of the oleogels developed (TFO: tallow fat oleogel, HTFO: hydrolized tallow fat oleogel, MGO: monoglyceride oleogel, MDGO: monoglyceride + diglyceride oleogel) 

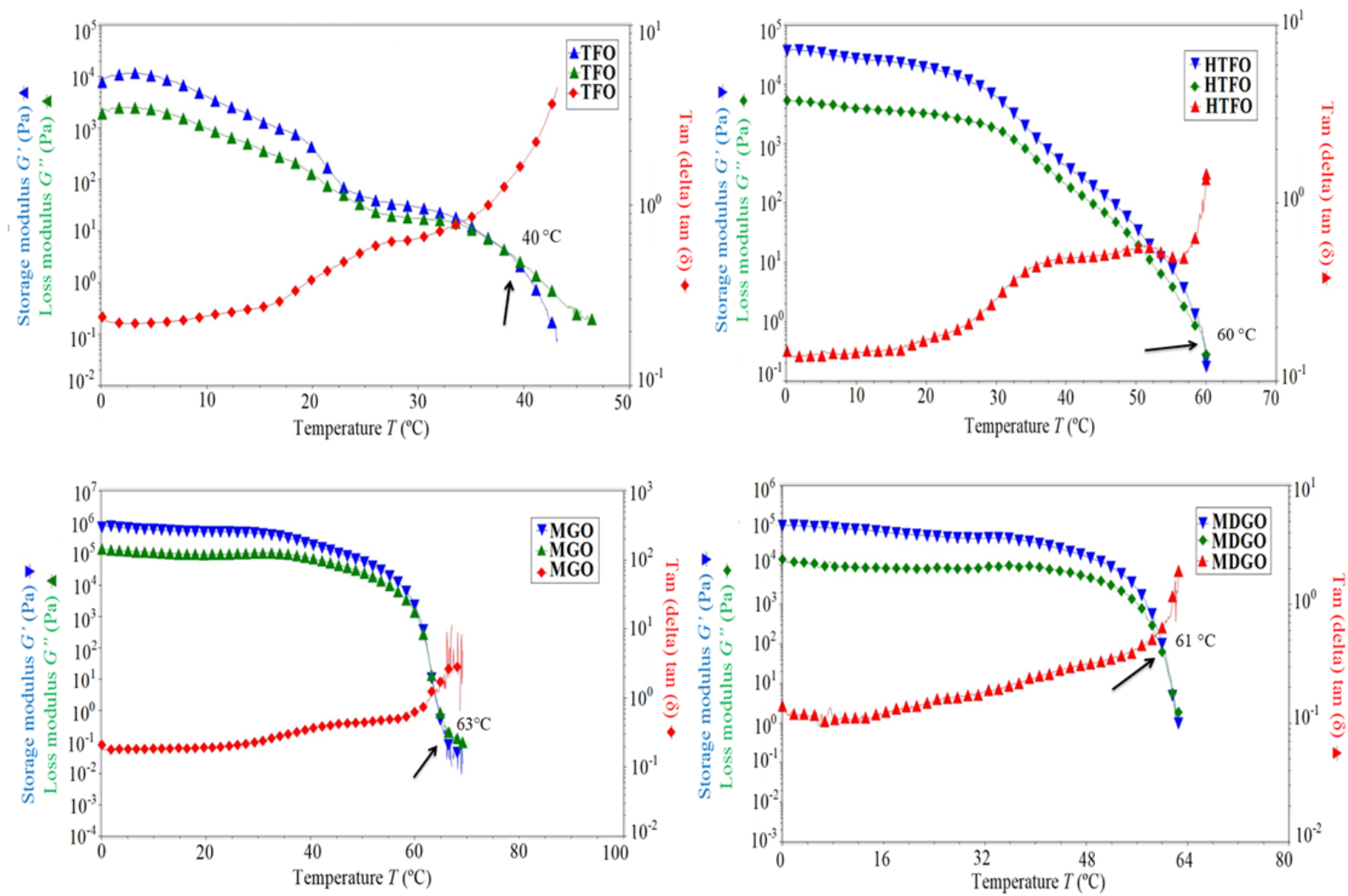

FIGURE 6. The temperature ramp test results of the oleogels developed (TFO: tallow fat oleogel, HTFO: hydrolized tallow fat oleogel, MGO: monoglyceride oleogel, MDGO: monoglyceride + diglyceride oleogel)

\section{ACKNOWLEDGMENTS}

This research was funded by the Scientific and Technological Council (TÜBİTAK) of Turkey with research Project no: 2170094, which we gracefully thank for the support.

\section{REFERENCES}

AOCS. 1998. Official Methods and Recommended Practices of the American Oil Chemists' Society, 5th Edn, AOCS Press, Champaigne 1998, IL.

Bot A, Veldhuizen YSJ, den Adel R, 2009. NonTAG structuring of edible oils and emulsions. Food Hydrocol. 23, 1184-1189. https://doi.org/ 10.1016/j.foodhyd.2008.06.009

Bot A, Flöter E. 2018. Structuring edible oil phases with fatty acids and alcohols, in Ashok R. Patel (Ed.), Edible Oil Structuring: Concept, Methods and Applications, Royal Soc. Chem., Chambridge, UK, 95-105.

Cerqueira MA, Fasolin LH, Picone CSF, Pastrana LM, Cunha RL, Vicente AA. 2017. Structural and mechanical properties of organogels: role of oil and gelator molecular structure. Food Res. Intl. 96, 161-170. https://doi.org/10.1016/ j.foodres.2017.03.021

Chrysam MM. 1996. Margarines and Spreads, in Y.H. Hui (Ed.), Bailey's Industrial Oil \& Fat Products, Vol. 3, Wiley-Intersience Pub, New York, US, 65-114.

Co ED, Marangoni AG. 2012. Organogels: An alternative edible oil-structuring method. $J$. Am. Oil Chem. Soc. 89, 749-780. https:// doi.org/10.1007/s11746-012-2049-3

Codex. 2017. Codex Alimentarius, International Food Standards, Standard for named vegetable oils, Codex Stan 210-1999.

Da Pieve S, Calligaris S, Co E, Nicoli MC, Marangoni AG. 2010. Shear nanostructuring of monoglyceride organogels. Food Biophys. 5, 211-217. https://doi.org/10.1007/s11483-010-9 $162-3$

Dassanayake LSK, Kodali DR, Ueno S. 2011. Formation of oleogels based on edible lipid materials. Current Op. Coll. Inter. Sci. 16, 432-439. https://doi.org/10.1016/j.cocis.2011. 05.005 
Dassanayake LSK, Kodali DR, Ueno S, Sato K. 2009. Physical properties of rice bran wax in bulk and organogels. J. Am. Oil Chem. Soc. 86, 1163-1173. https://doi.org/10.1007/s11746-009 $-1464-6$

Huang X, Wang J, Bi J, Hao H. 2018. Influence of diacylglycerol on physicochemical properties and crystallization behavior of palm oil. Chem. Eng. Technol. 41, 1587-1593. https://doi.org/10.1002/ceat.201700550

Kesselman E, Shimoni E. 2007. Imaging of oil/ monoglyceride networks by polarizing nearfield scanning optical microscopy. Food Biophys. 2, 117-123. https://doi.org/10.1007/ s11483-007-9038-3

Love JA. 1996. Animal fats, in Y.H. Hui (Ed.), Bailey's Industrial Oil \& Fat Products, Vol. 1, Wiley-Intersience Pub, New York, US, 1-18.

Mattice KD, Marangoni AG. 2018. New insights into wax crystal networks in oleogels, in Ashok R. Patel (Ed.), Edible Oil Structuring: Concept, Methods and Applications, Royal Soc. Chem., Chambridge, UK, 71-94.

Minitab. 2010. Minitab Statistical Software (Version 16.1). Minitab, Inc., State College, Pennsylvania, US.

ISO. 2008. Animal and vegetable fats and oilsDetermination of solid fat content by pulsed NMR-Part 2: Indirect method. ISO/TC 34/SC 11 , On-line version.

O'Brien RD. 2008. Fats and Oils: Formulating and Processing for Applications. CRC Press, New York, US.

Öğütcü M, Y1lmaz E. 2015. Characterization of hazelnut oil oleogels prepared with sunflower and carnauba waxes. Int. J. Food Prop. 18, 1741-1755. https://doi.org/10.1080/10942912. 2014.933352

Patel AR, Dewettinck K. 2015. Comparative evaluation of structured oil systems: shellac oleogel, HPMC oleogel and HIPE gel. Eur. J. Lipid Sci. Technol. 117, 1772-1781. https:// doi.org/10.1002/ejlt.201400553

Patel AR, Dewettinck K. 2016. Edible oil structuring: an overview and recent updates. Food Func. 7, 20-29. https://doi.org/10.1039/ $\mathrm{C} 5 \mathrm{FO} 01006 \mathrm{C}$

Patel AR. 2017. Methylcellulose-coated microcapsules of palm stearine as structuring templates for creating hybrid oleogels. Mater. Chem. Phys. 195, 268-274. https://doi.org/ 10.1016/j.matchemphys.2017.03.059

Patel AR. 2018. Oil structuring: concepts, overview and future perspectives, in Ashok R.
Patel (Ed.), Edible Oil Structuring: Concept, Methods and Applications, Royal Soc. Chem., Chambridge, UK, 3-24.

Perez-Monterroza EJP, Marquez-Cardozo CJ, Ciro-Velasquez HJ. 2014. Rheological behavior of avocado (Persea americana Mill, cv. Hass) oleogels considering the combined effect of structuring agents. LWT - Food Sci. Technol. 59, 673-679. https://doi.org/10.1016/ j.lwt.2014.07.020

Rocha-Amador OG, Gallegos-Infante JA, Huang Q, Rocha-Guzman NE, Moreno-Jimenez MR, Gonzalez-Laredo RF. 2014. Influence of commercial saturated monoglyceride, mono-/ diglycerides mixtures, vegetable oil, stirring speed, and temperature on the physical properties of organogels. Int. J. Food Sci. 513641，1-8. https://doi.org/10.1155/2014/513 641

Rogers MA. 2009. Novel structuring strategies for unsaturated fats - Meeting the zero-trans, zero-saturated fat challenge: A review. Food Res. Int. 42, 747-753. https://doi.org/10.1016/ j.foodres.2009.02.024

Sagiri SS, Samateh M, John G. 2018. Biobased molecular structuring agents, in Ashok R. Patel (Ed.), Edible Oil Structuring: Concept, Methods and Applications, Royal Soc. Chem., Chambridge, UK, 25-52.

Singh A, Auzanneau FI, Rogers MA. 2017. Advances in edible oleogel technology - a decade in review. Food Res. Int. 97, 307-317. https://doi.org/10.1016/j.foodres.2017.04.022

Stahl MA, Buscato MHM, Grimaldi R. 2017. Low sat-structured fats enriched in $\alpha$-linolenic acid: physicochemical properties and crystallization characteristics. J. Food Sci. Technol. 54, 3391-3403. https://doi.org/10.100 7/s13197-017-2780-8

Szydłowska-Czerniak A, Karlovits G, Lach M, Szłyk E. 2005. X-ray diffraction and differential scanning calorimetry studies of $\beta$ '$\beta$ transitions in fat mixtures. Food Chem. 92, 133-141. https://doi.org/10.1016/j.foodchem. 2004.07.010

Terech P, Weiss RG. 1997. Low molecular mass gelators of organic liquids and the properties of their gels. Chem. Rev. 97, 3133-3159. https:// doi.org/10.1021/cr9700282

Toro-Vasquez JE, Charo-Alonso MA, AlvarezMitre, FM. 2018. Gelation properties of gelator molecules derived from 12-hydroxystearic acid, in Ashok R. Patel (Ed.), Edible Oil Structuring: Concept, Methods and 
Applications, Royal Soc. Chem., Chambridge, UK, 106-134.

Viriato RLS, Queiros MS, da Gama MAS, Ribeiro APB, Gigante ML. 2018. Milk fat as a structuring agent of plastic lipid bases. Food Res. Int. 111, 120-129. https://doi.org/10.1016/ j.foodres.2018.05.015
Yılmaz E, Öğütcü M, Güneser O. 2015. Influence of storage on physico-chemical and volatile features of enriched and aromatized wax organogels. J. Am. Oil Chem. Soc. 92, 1429-1443. https://doi.org/10.1007/s11746-01 $5-2719-z$ 